\title{
SEMI-AUTOMATED ESTIMATION OF THE LOCAL FLOOD DEPTH ON SAR IMAGES
}

\author{
Abdelhakim Benoudjit and Raffaella Guida
}

\author{
Surrey Space Centre, University of Surrey \\ Guildford GU2 7XH, U.K. \\ a.benoudjit@surrey.ac.uk
}

\begin{abstract}
In the context of a flooding, a clear cloud-free SAR (Synthetic Aperture Radar) image proves mainly useful to retrieve flood features that can provide an extensive understanding of the disaster. Among these features, extremely important is the water depth on which this paper will focus by looking for a semi-automated algorithm for its estimation in the neighborhood of a given building from a pair of SAR images.

In this study, two SAR images acquired during dry and flooded conditions are necessary, as well as a DSM (Digital Surface Model) to give an a priori knowledge of the height of the building and its footprint. The whole process is divided into two main parts: First, an extraction of the building's double-bounce contribution using Genetic Algorithms, then the computation of the inundated building's height, to eventually evaluate the water level locally in the neighborhood of this building.

Thanks to the semi-automation of the double-reflection line retrieval, the execution time of the whole process was reduced from a few minutes (time to manually delineate the double-bounce line) to a few seconds, while keeping an error in the estimated flood depth in the order of a few decimeters $(35 \mathrm{~cm}$ on average).
\end{abstract}

Index Terms - Building detection, feature extraction, floods, synthetic aperture radar (SAR), urban areas

\section{INTRODUCTION}

The climate change is causing a rise in the number of floods over the years, which is predicted to grow even further in the future [1]. According to [2], floods were the most recurrent type of natural hazards in 2015 (around 40\% of the occurrences). This number is in agreement with those reported in [1] in the last two decades (1995 - 2015), when the flooding was the weather-related disaster that affected the most people worldwide ( 2.3 billion people excluding the deaths), and caused massive human deaths, with 157,000 casualties, in the same period.

\footnotetext{
This work was funded by SSTL.
}

During flood hazards, the affected area is characterized by cloud coverage which hampers the use of optical imagery for the purpose of mapping inundations. SAR (Synthetic Aperture Radar) sensors, on the other hand, thanks to their allweather and day-and-night capabilities, guarantee a monitoring of the disaster despite of the atmospheric or light conditions, which is particularly vital in urban settlements where the damages are the most substantial. Normally, buildings in urban areas lead to a rising double-backscatter intensity in SAR images, due to the dihedral surface they form [3]. However, the change in the urban radar return caused by the flood is hard to accurately measure, due to its dependency on various parameters (e.g. the building's aspect angle [4]).

In the majority of previous studies working with SAR images for the detection of floods, the inundation extent is essentially the only information extracted. Although, for certain applications like the assessment of the damages caused, additional inundation characteristics are needed to give a thorough analysis of the inundation hazard, like the water level [5]. In this context, the major advantage space-borne earth acquisitions have over gauging stations is the global-availability and the spatial-continuity of their data.

In this paper, the principal objective is to improve the work done in [6] by semi-automating the retrieval of the double-bounce contribution of a given building from the SAR image, before the subsequent estimation of the water level in its vicinity. The need for automation is motivated by the fact that automated inundation detection methods outperform the cumbersome and subjective manual flood mapping performed by human experts, especially in the aspect of the mapping speed [7], which is crucial to allow the civil protection authorities to react promptly with the adequate relief efforts.

\section{URBAN BACKSCATTERING MODEL}

In [8], an electromagnetic model was proposed to represent the backscattering from an isolated building on a rough ground surface. In this model, the intensity of the double-bounce, which is the predominant contribution for a 
parallelepiped-shaped building on a SAR image, can analytically be written in closed form by applying the Geometric Optics (GO) approximation for both bounces [9]:

$$
\begin{gathered}
\sigma^{0}=h \cdot f(p) \\
f(p)=\frac{\left|S_{p q}\right|^{2} l \tan \theta \cos \phi\left(1+\tan ^{2} \theta \sin ^{2} \phi\right)}{8 \pi^{2} \sigma^{2}\left(2 / L^{2}\right) \cos ^{2} \theta} \\
\exp \left[\frac{-\tan ^{2} \theta \sin ^{2} \phi}{2 \sigma^{2}\left(2 / L^{2}\right)}\right]
\end{gathered}
$$

where the two variables relevant to this study are:

$$
\begin{aligned}
& \sigma^{0} \text { : The intensity of the building's double-bounce, } \\
& h \text { : The building's height. }
\end{aligned}
$$

The formula in (1) was inverted in [9] to estimate the height of a building from the intensity of its double-bounce contribution on the SAR image, by assuming that the dielectric and roughness properties of the scene materials were given or had been measured a priori. The same rationale was applied in [6] to evaluate, this time, the flood depth on SAR images, by considering the soil flooded and changing its physical and electrical properties in (2) accordingly. The strength of this method lies in the fact that only a single SAR image is needed for the estimation of the building's height, without the necessity for any additional ancillary data. Besides, the error yielded in the height estimation should be, in theory, smaller than the spatial resolution, which cannot be achieved with methods based on counting the number of pixels an image feature is made up of [9].

\section{METHODOLOGY}

The tasks described in the following sections are part of a sequential process that gives the local flood depth near a building of interest in a semi-automated fashion, knowing the height of this same building, as shown in the flowchart in Figure 1. In the flowchart, both the extraction of the building's double-backscatter contribution and the estimation of the building's height rely on the urban backscattering model given in the previous section. Moreover, the same values for the roughness and dielectric properties of the ground and the building's wall materials measured in situ in [6] were used here, thanks to the fact that the flood level estimation was performed on the same dataset.

\subsection{Coregistration of SAR images with the DSM}

Initially, two SAR images taken before and after the flood were automatically coregistered in SNAP (Sentinel Application Platform) [10] with the LiDAR DSM, which was chosen as a master thanks to its higher spatial resolution. The coregistration is necessary to locate the building footprint on

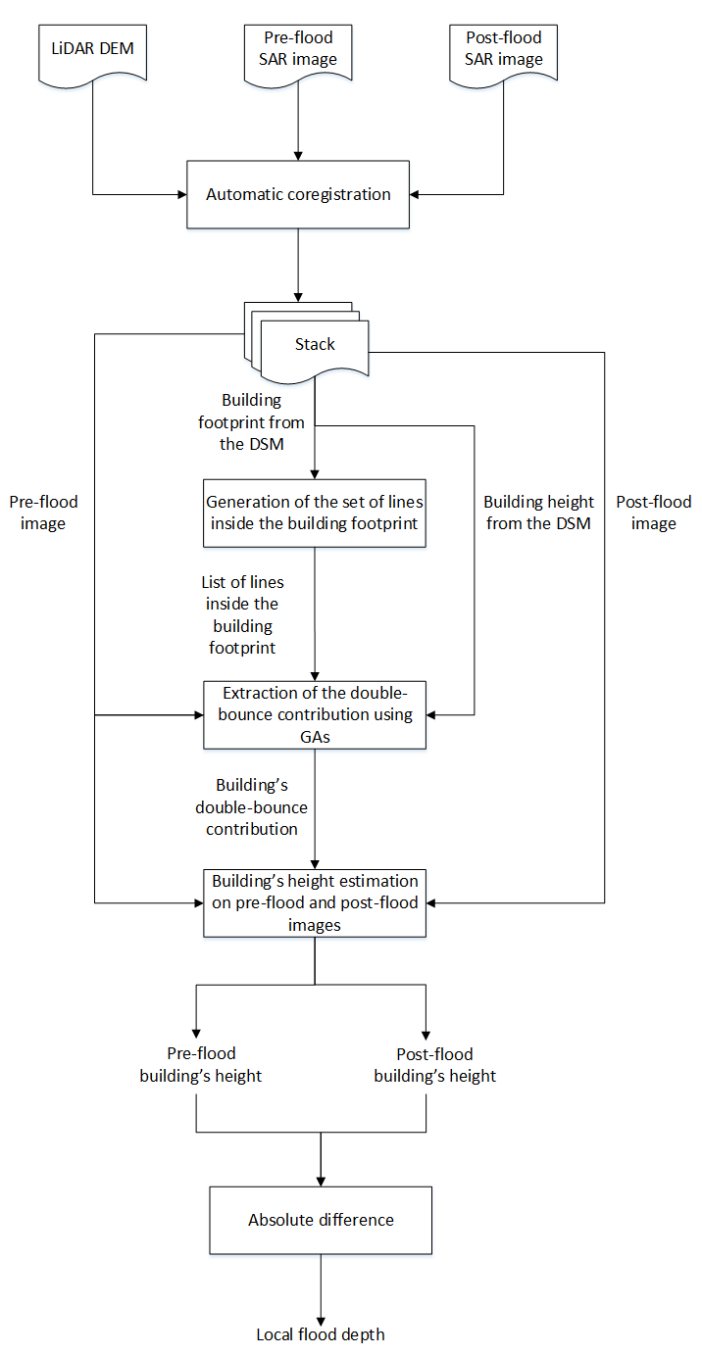

Fig. 1. Flowchart of the proposed semi-automated local flood depth estimation process.

SAR images (Red rectangle in Figures 3-a and 3-b), from its position on the LiDAR DSM (Red rectangle in Figure 3-c). The subsequent algorithm will look for the building's doublebounce contribution inside the red rectangle on the pre-flood SAR image (Figure 3-a). Prior to the double-bounce extraction, the footprint was expanded to contain the entire doublebounce contribution, which is distorted on SAR images because of two reasons: 1) the building of interest is not parallel to the radar flight direction and 2) the sensor operates in a side looking configuration.

\subsection{Extraction of the building's double-bounce}

The goal of this experiment is to reduce the errors in the building height estimated from the inversion of the previous urban backscattering model. These errors arise from the difficulty to determine the pixels that truly belong to the double-bounce 
contribution relevant to a specific building on the pre-flood SAR image.

First, it was assumed that the height of the building in normal conditions, as well as its footprint can be both retrieved from a high-resolution LiDAR DSM (Digital Surface Model) of the same area, and used as a prior knowledge during the double-bounce extraction. Then following the method described hereinafter, it was possible to extract the building's double-bounce contribution, from the pre-flood SAR image, for which the average backscatter provides the closest height estimation relatively to the one known from the LiDAR DSM. This method is based on GAs (Genetic Algorithms) [11] and was implemented using the DEAP (Distributed Evolutionary Algorithms in Python) framework [12].

The double-bounce extraction from the pre-flood SAR image, described below, depends on the accurate coregistration between LiDAR and SAR images, to ensure that the targeted double-bounce contribution in the SAR image is located inside the building's LiDAR footprint (theoretically, it should appear precisely on the footprint edge). The set of genes (blue lines consisting of yellow pixels in Figure 2-a) is generated inside the building footprint (Red rectangle in Figure 2-a) using the DDA (Digital Differential Analyzer) algorithm [13], which is intended to rasterize lines. The latter algorithm is, firstly, employed to link the four corner pixels of the building footprint. Then, using the same method, the blue lines (genes) are drawn inside the building footprint from yellow pixels on one thick red line to the pixels facing them on the opposite thick red line (See Figure 2-a).

The double-bounce candidates (called chromosomes in the GA terminology) generated afterwards are made up of a specific number of adjacent footprint blue lines (See the generated green and blue chromosomes in Figure 2-b). These chromosomes were created by slicing the list of genes starting from a random position (i.e. random blue line in Figure 2-a), and ending a few lines later (according to the number of lines per chromosome). Genetic Algorithms, in general, work by iteratively optimizing the fitness of the chromosomes in the population. In our particular case, we aim to minimize the chromosome's fitness value, which is simply calculated in (3) as the absolute difference between the chromosome's height estimated by inverting (1), and the expected building height extracted from the LiDAR elevation data. During the estimation of the height of a chromosome, the average backscatter of the pixels (yellow dots in Figure 2-b) composing it on the preflood SAR image, was considered as the backscatter $\sigma^{0}$ of the whole chromosome in (1). After that, the fitness parameter was worked out as follows:

$$
\text { Fitness }=\left|h_{\text {estimated }}-h_{\text {expected }}\right|
$$

where:

Fitness: The fitness of the chromosome,

$h_{\text {estimated }}$ : The estimated height of the chromosome. $h_{\text {expected }}$ : The building height measured by the LiDAR DSM.

The population of chromosomes produced in the previous step is evolved for a given number of generations, by performing three genetic operators on its individuals: selection, mutation, and crossover [11]. A tournament selection is carried out to select the fittest chromosomes in the population, that will be eligible to subsequently mutate and mate (crossover). This type of selection performs a series of independent tournaments between randomly drawn chromosomes, and chooses the fittest among the competitors every time. It thus does not necessarily select only the fittest chromosomes in the population, which is convenient to promote diversity in it.

Next, according to two arbitrary probabilities ( $p_{\text {mutation }}$ and $p_{\text {crossover }}$, respectively), a mutation is first performed on randomly-selected chromosomes to avoid getting stuck in a local minimum, followed by a crossover between two random chromosomes to produce new offspring. The mutation implemented consists in replacing a random pixel in the selected chromosome with another one chosen randomly from the two adjacent gene lines (See the added yellow dots in the adjacent purple lines in Figure 2-c). Subsequently, a single-point crossover is carried out between the selected pair of individuals by swapping pixels situated after a random position (The crossover between chromosomes in Figure 2-b gives the offspring in Figure 2-d). Eventually, the parents will be replaced in the population by their offspring, for which the fitness is re-evaluated.

\subsection{Building's height estimation}

In the following, the fittest individual produced in the last generation of the previous step is considered in the height estimation phase, which exploits the formula given in (1). The heights of this individual prior to and after the inundation were estimated from its mean intensity in the pre-flood and the post-flood SAR images, respectively, by inverting (1) [9].

\subsection{Estimation of the local flood depth}

Ultimately, the water level was calculated, straightforwardly, as the absolute difference between the building heights in normal and flooded conditions [6].

\section{CASE STUDY AND DATASET}

\subsection{Dataset}

The Gloucestershire flooding event (UK) in the summer of 2007, which came as a consequence of the record-breaking heavy rainfall between the months of May and July, claimed 13 lives and has been the costliest hydrological disaster in the same year worldwide [14]. 


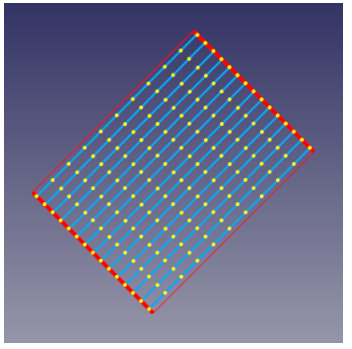

(a)

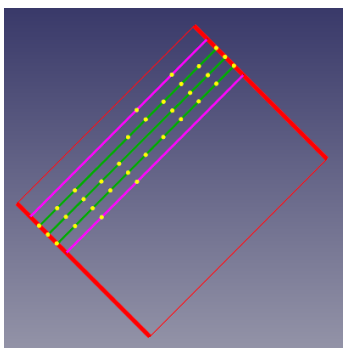

(c)

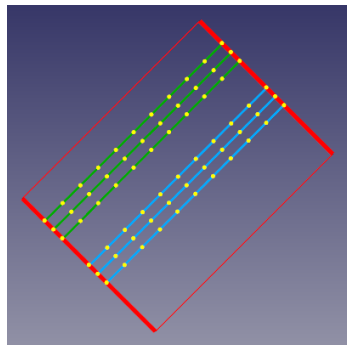

(b)

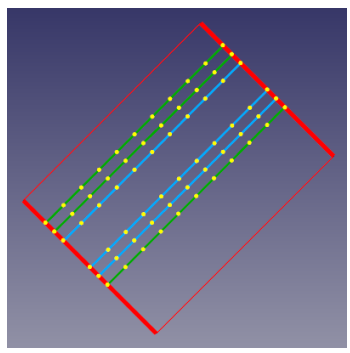

(d)
Fig. 2. (a) Set of genes (blue lines consisting of yellow pixels) inside the building footprint (red rectangle) (b) Two chromosomes (double-bounce candidates) consisting of three genes (lines) (c) Mutation of a chromosome (Replacing random chromosome pixels with pixels from adjacent lines) (d) Crossover between two chromosomes (swapping of last lines of genes).

TerraSAR-X, although still in its commissioning phase, acquired an $\mathrm{HH}$-polarized stripmap image ( $3 \mathrm{~m}$ resolution) of the flooded city of Tewkesbury (Gloucestershire) on the 25th of June 2007 (Figure 3-b), which is distributed as a SSC (Single Look Slant Range Complex) product. Another SAR image was acquired by TerraSAR-X a year later (22nd of June 2008 ) in the same configuration, and is considered the preflood image (Figure 3-a) in what follows.

Airborne LiDAR surveys were carried out in the last 17 years across England to capture the terrain elevation with a resolution ranging from $2 \mathrm{~m}$ to $25 \mathrm{~cm}$. This data is freelydistributed [15] either as a DSM (Digital Surface Model) (Figure 3-c) or as a DTM (Digital Terrain Model), which is basically a DSM where the vegetation and buildings were flatten to leave only the bare-earth.

\subsection{Preprocessing}

Initially, the LiDAR DSM elevation data was re-projected from the British National Grid projection (its original projection) to the standard WGS84 (World Geodetic System) projection. As for the pre-flood and post-flood SAR SSC (Single Look Slant Range Complex) products, they were calibrated and projected from the slant-range to the ground-range geometry by performing a terrain correction using, this time,

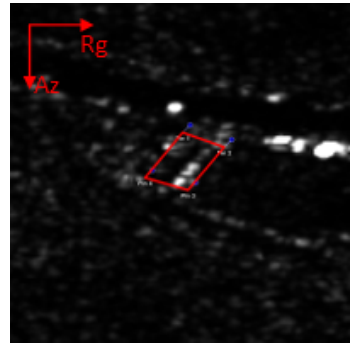

(a)

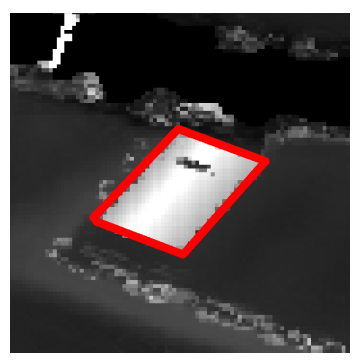

(c)

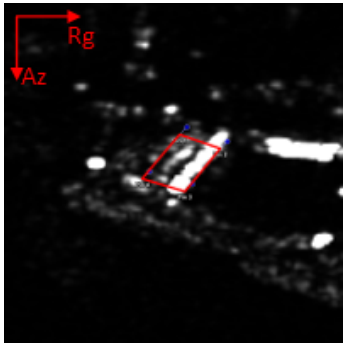

(b)

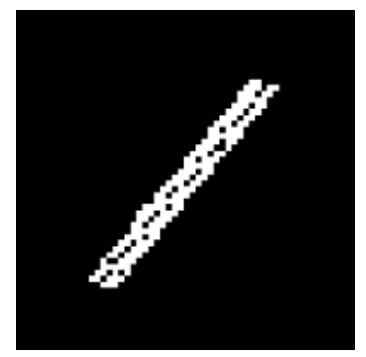

(d)
Fig. 3. Subset of the city of Tewkesbury including the building of interest (inside the red rectangle) on (a) the TerraSAR$X$ pre-flood image (b) the TerraSAR-X post-flood image (c) the LiDAR DSM. (d) The double-bounce contribution extracted from the pre-flood SAR image.

the DTM.

\section{RESULTS}

The water level of the Severn River as measured by the Mythe Bridge water gauge was used as a ground truth to assess the results of the method proposed. However, this water gauge is located less than a kilometer away from the building-ofinterest, which is itself situated at the junction of the River Avon and the River Severn (Figure 3). The distance between the Mythe Bridge station and the studied building might invalidate using this water gauge as a ground truth due to the fluctuation in the river level. Moreover, it is worth mentioning that only a visual estimation of the river embankment near the building was given in [6], while no actual measurement was carried out. The error in the water level estimated with our algorithm relatively to the ground truth is on average equal to $35 \mathrm{~cm}$ with a standard deviation of $40 \mathrm{~cm}$ (See Table 1), if we consider an embankment of $1.5 \mathrm{~m}$ as in [6], while the error obtained in the latter paper was $24 \mathrm{~cm}$. The mean and standard deviation of the error in the flood depth estimated were obtained in our experiment after executing our program 100 times.

The estimated building heights and the water depth shown in the first row of Table 1, were obtained for the extracted building's double-bounce contribution shown in Figure 3-d. 


\begin{tabular}{|c|c|c|c|c|}
\hline & $\begin{array}{c}\text { Pre- } \\
\text { flood } \\
\text { height } \\
{[\mathrm{m}]}\end{array}$ & $\begin{array}{c}\text { Post- } \\
\text { flood } \\
\text { height } \\
{[\mathrm{m}]}\end{array}$ & $\begin{array}{c}\text { Water } \\
\text { depth } \\
{[\mathrm{m}]}\end{array}$ & $\begin{array}{c}\text { Error } \\
{[\mathrm{m}]}\end{array}$ \\
\hline $\begin{array}{c}\text { This } \\
\text { paper's } \\
\text { results }\end{array}$ & 5.53 & 3.02 & 2.51 & $\begin{array}{c}\text { Avg: } 0.35 \\
\text { Std: } 0.40\end{array}$ \\
\hline $\begin{array}{c}{[6] \text { 's }} \\
\text { results }\end{array}$ & 5.53 & 2.57 & 2.96 & 0.24 \\
\hline $\begin{array}{c}\text { Ground } \\
\text { truth [6] }\end{array}$ & - & - & 2.72 & - \\
\hline
\end{tabular}

Table 1. The estimated flood depth near the building-ofinterest compared to [6] and to the ground truth.

In order to compare our results to those obtained in [6], the $a$ priori known building height $(5.53 \mathrm{~m})$ was taken from the latter paper. Errors in the estimated flood depth might be caused by an inaccurate automatic coregistration, which could create a shift in the position of the double-bounce contribution between pre-flood and post-flood SAR images.

Because our algorithm is stochastic (chromosomes are generated randomly), different executions will not necessarily produce the exact same flood depth value. This explains why the standard deviation of the error was larger than its mean (i.e. due to outliers in the distribution of the flood depth error). However, the processing time required to estimate the local flood height in the vicinity of one building using a GA run for 300 generations was virtually steady, and on average less than 10 seconds. These results were obtained on a workstation equipped with an Intel Xeon X5650 CPU with 24GB of RAM. On a more powerful machine (e.g. a cluster), the results will certainly improve in terms of speed, which opens the possibility to estimate the flood depth on a larger scale (i.e. on the whole image), especially if multiple calls to our algorithm (flood depths estimated near multiple buildings) are made in parallel.

It should take several minutes for a human operator to manually extract one double-bounce contribution. During the same period of time, a scaled version of the method discussed in this paper would be able to give an estimation of the water level near multiple buildings (following the trends given above), which could be interpolated to provide emergency services with a 3D visualization of the flooding event.

\section{CONCLUSION}

In summary, a semi-automated process was proposed in this paper to give the flood depth locally in the vicinity of an inundated building from a pair of high-resolution SAR images using a genetic algorithm followed by the inversion of an urban backscattering model. One potential application of this method is to assist the insurance companies in the assessment of the damages incurred by buildings and structures in flooded urban areas. Nevertheless, the estimation of the water level is dependant on the availability of a high-resolution LiDAR DSM, which is not guaranteed worldwide especially for poor countries.

\section{REFERENCES}

[1] Center for Research on the Epidemiology of Disasters (CRED) \& The United Nations Office for Disaster Risk Reduction (UNISDR), "The human cost of weather related disasters (1995-2015)," Brussels: CRED, 2015.

[2] D. Guha-Sapir, Ph. Hoyois, and R. Below, "Annual Disaster Statistical Review 2015: The Numbers and Trends," Brussels: CRED, 2016.

[3] M. Watanabe, M. Shimada, M. Matsumoto, and M. Sato, "GB-SAR/PiSAR simultaneous experiment for a trial of flood area detection," 2008 IEEE International Geoscience and Remote Sensing Symposium (IGARSS), pp. 27-30, 2008.

[4] A. Ferro, D. Brunner, L. Bruzzone, and G. Lemoine, "On the Relationship Between Double Bounce and the Orientation of Buildings in VHR SAR Images," IEEE Geoscience and Remote Sensing Letters, vol. 8, no. 4, pp. 612-616, 2011.

[5] H. Zwenzner and S. Voigt, "Improved estimation of flood parameters by combining space based SAR data with very high resolution digital elevation data," $H y$ drology and Earth System Sciences Discussions, vol. 5, no. 5, pp. 2951-2973, 2008.

[6] P. Iervolino, R. Guida, A. Iodice, and D. Riccio, "Flooding Water Depth Estimation With High-Resolution SAR," IEEE Transactions on Geoscience and Remote Sensing, vol. 53, no. 5, pp. 2295-2307, 2015.

[7] S. Martinis, a. Twele, and S. Voigt, "Towards operational near real-time flood detection using a splitbased automatic thresholding procedure on high resolution TerraSAR-X data," Natural Hazards and Earth System Science, vol. 9, no. 2, pp. 303-314, 2009.

[8] G. Franceschetti, A. Iodice, and D. Riccio, "A canonical problem in electromagnetic backscattering from buildings," IEEE Transactions on Geoscience and Remote Sensing, vol. 40, no. 8, pp. 1787-1801, 2002.

[9] R. Guida, A. Iodice, and D. Riccio, "Height Retrieval of Isolated Buildings From Single High-Resolution SAR Images," Geoscience and Remote Sensing, IEEE Transactions on, vol. 48, no. 7, pp. 2967-2979, 2010. 
[10] European Space Agency (ESA), "Sentinel Application Platform (SNAP),"

http://step.esa.int/main/toolboxes/

snap/, 2016, [Online; accessed 22-November-2016].

[11] Melanie Mitchell, An introduction to genetic algorithms, MIT press, 1996.

[12] F.M. De Rainville, F.A. Fortin, M.A Gardner, M. Parizeau, and Ch. Gagné, "DEAP : A Python Framework for Evolutionary Algorithms," Companion proc. of the Genetic and Evolutionary Computation Conference, pp. 85-92, 2012.

[13] Alan Watt, 3D computer graphics, Addison-Wesley, 3rd edition, 2000.

[14] M. Pitt, "Learning Lessons from the 2007 Floods," The Pitt Review, 2008.

[15] Data.gov.uk, "LiDAR Digital Elevation Model (DEM) of the city of Tewkesbury,"

http://environment.data.gov.uk/ds/ survey/index. jsp\#/ survey?grid=S083, 2016, [Online; accessed 21-November-2016]. 